\title{
Alfvén instability of steady state flux tubes
}

\section{Upflows in stratified atmospheres}

\author{
Y. Taroyan \\ Department of Physics, IMPACS, Aberystwyth University, Penglais, Aberystwyth SY23 3BZ, UK \\ e-mail: yot@aber.ac.uk \\ Received 7 July 2014 / Accepted 21 January 2015

\begin{abstract}
Context. MHD instabilities play an important role in the dynamics and energetics of the solar atmosphere.

Aims. An open vertical magnetic flux tube is permeated by an upflow in a stratified atmosphere with variable temperature. The stability of the tube is investigated with respect to small-amplitude torsional perturbations generated at the footpoint by random convective motions.

Methods. A steady state equilibrium incorporating the effects of a vertical body force, heating, and losses is derived analytically. The governing equations for torsional motions are integrated with a fourth-order Runge-Kutta method and matched with the analytical solutions in the upper regions to obtain a numerical dispersion relation. The dependence of the eigenmode frequencies on different parameters is analysed. Unstable modes are found for a range of Alfvén and flow speeds in the photosphere, as well as expansion factors of the flux tubes. Both supersonic and subsonic flows are considered.

Results. It is shown that torsional perturbations are exponentially amplified in time if a section of the tube exists where the upflowing plasma decelerates and the tube expands. The flow speeds required for the instability are sub-Alfvénic.

Conclusions. The instability may be important for understanding the abundance of Alfvén waves seen in recent observations and the associated heating in magnetic regions of the solar atmosphere.
\end{abstract}

Key words. instabilities - magnetohydrodynamics (MHD) - waves - Sun: atmosphere

\section{Introduction}

Since their discovery in the 1940s Alfvén waves have been studied in relation to the heating of laboratory plasmas and the solar atmosphere, the formation of spicules, and the acceleration of the solar wind. The waves result from the competing effects between magnetic tension and plasma inertia. Alfvén waves are notoriously difficult to detect since there are no associated variations in density or the field strength. Recent observations have shown the abundance of transverse and torsional waves in various structures of the solar atmosphere (Nakariakov et al. 1999; Tomczyk et al. 1998). The waves observed in the lower atmosphere are usually associated with field-aligned upflows (Peter 2000; Xia et al. 2003; Hara et al. 2008; Jess et al. 2009; De Pontieu et al. 2012; Morton et al. 2012). Estimates of the energy flux carried by the waves indicate that they could power the solar wind and heat the corona (McIntosh et al. 2011). A theoretical and observational overview of Alfvén waves in various structures of the solar atmosphere is presented by Mathioudakis et al. (2013).

Any theory dealing with the energetic implications of Alfvén waves should consider their generation, propagation, and dissipation as equally important aspects of the same problem. However, such a unified treatment has proved to be a challenge.

Various models have been employed to explain the generation of waves, the energy transfer, and the dissipation in the atmosphere. Parker (1991) argues that photospheric convection is unlikely to produce Alfvén waves with sufficiently large amplitudes to heat the corona or to power the solar wind, nor would such waves dissipate significantly in the first couple of solar radii if they even existed. The generation of Alfvén waves is more efficient when the fibril structure of the photospheric field is taken into account but still not enough to provide adequate wave flux into the corona (Muller et al. 1994; Matsumoto \& Kitai 2010).

Compared to other types of waves, Alfvén waves are able to carry energy over long distances along magnetic field lines. They are least susceptible to shock formation and dissipation. However, reflections due to temperature increase and tube expansion are both possible (Murawski \& Musielak 2010; Routh et al. 2010). The reflections may also lead to resonant cavities (Hollweg 1984; Matsumoto \& Shibata 2010).

Alfvén waves are able to dissipate their energy through various mechanisms, such as resonant absorption, nonlinear coupling to slow and fast MHD shocks, and phase mixing (Ionson 1978; Hollweg et al. 1982; Heyvartes \& Priest 1983). Parker (1991), Ofman \& Davila (1995) argues that in open structures dissipation due to phase mixing is expected to occur only within several solar radii. Belien et al. (1999) find that the efficiency of resonant absorption can be very low owing to the fast rate at which slow magnetosonic waves are nonlinearly generated in the chromosphere and transition region. This leads to considerable transfer of energy from the Alfvén wave to the magnetosonic waves. Antolin \& Shibata (2008) find that the regimes under which Alfvén wave heating produces hot and stable coronae are rather narrow: independently of the photospheric wave amplitude and magnetic field, a corona can be produced and maintained only for long (>80 Mm) and thick loops. 
Magnetohydrodynamic (MHD) instabilities play a key role in a number of processes occurring in the Sun and in the solar-terrestrial environment: small perturbations become exponentially amplified, leading to large scale changes in the system. Well-known examples include the Rayleigh-Taylor and the Kelvin-Helmholtz instabilities.

Taroyan (2008) established the possibility of a new MHD instability associated with incompressible Alfvénic disturbances in compressible plasma flows. It does not require a shear and may arise at rather moderate sub-Alfvénic flow speeds owing to the compressibility of the plasma flow. In a two-layer semi-infinite model, small-amplitude Alfvénic disturbances become exponentially amplified because they subtract energy from the flow and become over-reflected - a concept introduced by Acheson (1976) in the context of Kelvin-Helmholtz instability. In the case of the Alfvén instability, a cavity is set up within which the Alfvénic perturbations grow as they bounce back and forth.

An application of the instability to coronal loops with siphon flows was presented by Taroyan (2009). It was shown that in asymmetric loops with siphon flows, linear torsional perturbations driven at the footpoints may become exponentially amplified for arbitrary flow speeds.

The magnetic field outside sunspots is concentrated in flux tubes with kilogauss field strengths and widths of a few hundred kilometers (Stenflo 1989). Gabriel (1976) argued that the expansion of flux tubes above the photosphere is so rapid that field lines at the edges are nearly horizontal, leading to a magnetic canopy at a height of about $500 \mathrm{~km}$. More recently, Tsuneta et al. (2008), Verth et al. (2011), and Morton et al. (2012) have explored the expansion of flux tubes above the photosphere at chromospheric heights.

Taroyan (2011) analysed the instability in gravitationally stratified expanding flux tubes. It was assumed that the equilibrium quantities, such as the flow and the magnetic field, are smooth functions of height. This analysis was limited to isothermal flows.

The present paper extends the analysis by Taroyan (2011) to a wider class of flux tubes with nonisothermal flows by including a full equation of energy and a body force in the momentum equation. The equilibrium is derived analytically; an analytical criterion for the instability is obtained; the behaviour in supersonic and subsonic flows is compared; rapidly and moderately expanding flux tubes are treated separately; the dependence of the instability on the expansion factor is analysed; and a schematic illustration of the instability conditions is presented in Fig. 8.

\section{Model and governing equations}

We use the axisymmetric magnetic flux tube model introduced by Hollweg et al. (1982). The distance along a single field line is denoted by $s$. The photospheric boundary is placed at $s=0$. The distance from the axis of symmetry is denoted by $r=r(s)$, i.e., any radial expansion or contraction of the flux tube in time are assumed to be negligible. The azimuthal angle about the axis of symmetry is denoted by $\theta$. In the azimuthal direction, only axisymmetric motions are considered, so $\partial / \partial \theta=0$.

The model was developed by different authors to include various source terms. In particular, the effects of heating, radiation, and conduction were first studied by Mariska \& Hollweg (1985), and the effects of a body force were originally discussed by Sterling \& Hollweg (1988). The model has been applied to both linear and nonlinear problems in various solar and stellar contexts (Sterling \& Hollweg 1984; Mariska \& Hollweg 1985; Kudoh \& Shibata 1997; Moriyasu et al. 2004; Fujita et al. 2007; Musielak et al. 2007; Antolin \& Shibata 2008).

With the assumptions made above the following nonlinear equations of conservation of mass, momentum, energy and induction for the mass density $\rho$, pressure $p$, the $s$ and $\theta$ components of the magnetic field, $\left(B_{s}, B_{\theta}\right)$, and velocity, $\left(u_{s}, u_{\theta}\right)$ are derived:

$$
\begin{aligned}
& \frac{\partial}{\partial t}\left(\frac{\rho}{B_{s}}\right)+\frac{\partial}{\partial s}\left(\frac{\rho u_{s}}{B_{s}}\right)=0, \\
& \frac{\partial}{\partial t}\left(\frac{\rho r u_{\theta}}{B_{s}}\right)+\frac{\partial}{\partial s}\left(\frac{\rho r u_{\theta}}{B_{s}} u_{s}\right)=\frac{1}{\mu_{0}} \frac{\partial}{\partial s}\left(r B_{\theta}\right), \\
& \frac{\partial}{\partial t}\left(\frac{\rho u_{s}}{B_{s}}\right)+\frac{\partial}{\partial s}\left(\frac{\rho u_{s}}{B_{s}} u_{s}\right)=-\frac{1}{B_{s}} \frac{\partial p}{\partial s}+\frac{\rho}{B_{s}}\left(g_{s}+\mathcal{F}\right) \\
& \quad+\frac{1}{B_{s}} \times\left[\left(\rho u_{\theta}^{2}-\frac{B_{\theta}^{2}}{\mu_{0}}\right) \frac{\partial \ln r}{\partial s}-\frac{\partial}{\partial s}\left(\frac{B_{\theta}^{2}}{2 \mu_{0}}\right)\right], \\
& \frac{\partial}{\partial t}\left(\frac{p}{B_{s}}\right)+\frac{\partial}{\partial s}\left(\frac{p}{B_{s}} u_{s}\right)=-(\gamma-1) p \frac{\partial}{\partial s}\left(\frac{u_{s}}{B_{s}}\right) \\
& \quad+\frac{\gamma-1}{B_{s}}\left[\mathcal{H}+\frac{1}{r^{2}} \frac{\partial}{\partial s}\left(r^{2} \kappa T^{5 / 2} \frac{\partial T}{\partial s}\right)-\mathcal{R}\right], \\
& \frac{\partial}{\partial t}\left(\frac{B_{\theta}}{r B_{s}}\right)+\frac{\partial}{\partial s}\left(\frac{B_{\theta}}{r B_{s}} u_{s}\right)=\frac{\partial}{\partial s}\left(\frac{u_{\theta}}{r}\right),
\end{aligned}
$$

where $g_{s}$ is the $s$ component of the gravitational acceleration, $\gamma$ is the adiabatic index, and $\mathcal{F}$ is a prescribed body force acting on the plasma. In the energy Eq. (4), the terms $\mathcal{H}, \mathcal{R}$ represent heating and radiative losses, respectively, while $\kappa$ is the coefficient of themral conduction. In the above equations, $B_{s}$ is a function of $s$ and does not depend on $t$. The condition for the conservation of magnetic flux can be reduced to

$B_{s}(s) r^{2}(s)=$ const.

provided the chosen field line is near the axis of the flux tube.

The body force term is borrowed from Sterling \& Hollweg (1988) who used this source term to model granular buffeting of a flux tube and the subsequent generation of spicules. In our study, the body force could be due to granular buffeting or any other mechanism which combined with the source terms in the energy equation supports the upflows.

No assumptions are made about the exact form of the radiative loss function or the phenomenological heating term $\mathcal{H}$. The following Eqs. (14)-(18) are derived for arbitrary radiative losses and heating which may also depend on density and temperature. The equilibrium quantities of interest are expressed in terms of the flow speed, temperature and the body force.

\subsection{Steady state}

The existence of a steady state equilibrium is determined by the body force $\mathcal{F}$ and the heating rate $\mathcal{H}$. An equilibrium will exist if $\mathcal{F}$ and $\mathcal{H}$ are time-independent. In a realistic solar atmosphere, the above assumptions can be justified if the latter two quantities change slowly compared to the wave period. The equilibrium structure of the flux tube is shown in Fig. 1. The equilibrium quantities are denoted by a subscript 0 . The magnetic field $\boldsymbol{B}_{0}$ is untwisted, i.e., only the $s$ component is present. The flux tube is permeated by a field-aligned mass flow $\boldsymbol{u}_{0}$. We consider field 


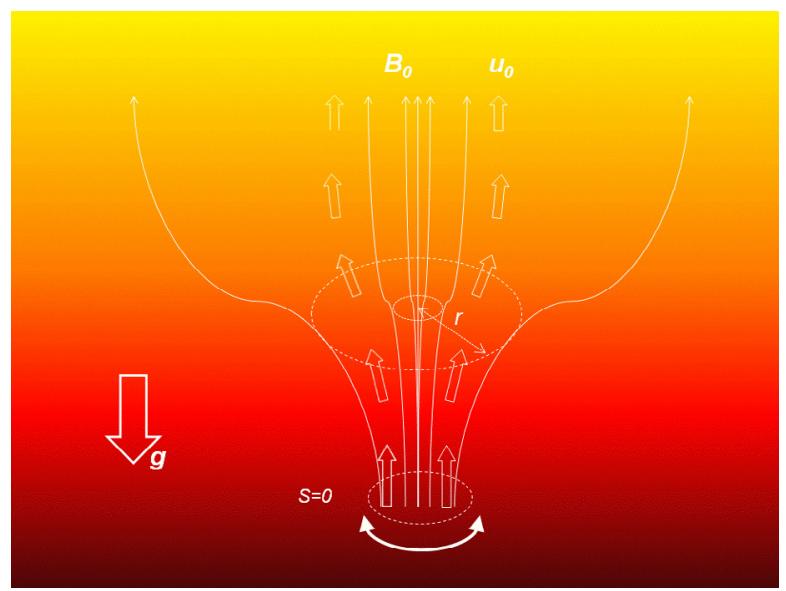

Fig. 1. Cartoon of an expanding flux tube in which plasma flows along the field lines. The $s=0$ level represents the footpoint which is twisted by convective motions.

lines near the tube axis for which $g_{s}=-g$ (Hollweg et al. 1982). The steady equilibrium is determined by the conservation equations of mass (1), momentum (3) and energy (4):

$\frac{\mathrm{d}}{\mathrm{d} s}\left(\frac{\rho_{0} u_{0}}{B_{0}}\right)=0$

$\frac{\mathrm{d}}{\mathrm{d} s}\left(\frac{\rho_{0} u_{0}}{B_{0}} u_{0}\right)=\frac{1}{B_{0}}\left(-\frac{\mathrm{d} p_{0}}{\mathrm{~d} s}-\rho_{0} g+\rho_{0} \mathcal{F}\right)$,

$\frac{\mathrm{d}}{\mathrm{d} s}\left(\frac{p_{0} u_{0}}{B_{0}}\right)=-(\gamma-1) p_{0} \frac{\mathrm{d}}{\mathrm{d} s}\left(\frac{u_{0}}{B_{0}}\right)$

$+\frac{\gamma-1}{B_{0}}\left[\mathcal{H}+\frac{1}{r^{2}} \frac{\mathrm{d}}{\mathrm{d} s}\left(r^{2} \kappa T_{0}^{5 / 2} \frac{\mathrm{d} T_{0}}{\mathrm{~d} s}\right)-\mathcal{R}_{0}\right]$,

where the subscript 0 denotes the corresponding equilibrium quantity along a field line.

According to the ideal gas law, pressure is determined through density and temperature. Therefore Eqs. (7)-(9) contain six unknowns: $\rho_{0}, T_{0}, u_{0}, B_{0}, \mathcal{H}, \mathcal{F}$. Three of those can be expressed in terms of the heating rate, $\mathcal{H}$, the body force, $\mathcal{F}$, and the magnetic field strength, $B_{0}$. Note that according to Eq. (6) the magnetic field is determined by the cross-sectional area of the axisymmetric flux tube since $B_{0}=B_{s}$. Thus the steady state is determined by the heat input and the shape of the flux tube. From a mathematical point of view, it is more convenient to derive analytical expressions for the equilibrium quantities in terms of the flow speed, $u_{0}$, the body force, $\mathcal{F}$, and temperature $T_{0}$. Equation (9) can be rewritten as

$\frac{1}{c_{s}^{2}} \frac{\mathrm{d} c_{s}^{2}}{\mathrm{~d} s}=\frac{\gamma-1}{\rho_{0}} \frac{\mathrm{d} \rho_{0}}{\mathrm{~d} s}+\frac{(\gamma-1) \gamma B_{0}}{\rho_{0} u_{0} c_{s}^{2}} \mathcal{S}$,

where $c_{\mathrm{s}}^{2}=\gamma p_{0} / \rho_{0}=$ const. $\times T_{0}$ is the sound speed and $\mathcal{S}$ is the sum of sources and sinks of energy in Eq. (9):

$\mathcal{S} \equiv \frac{1}{B_{0}}\left[\mathcal{H}+\frac{1}{r^{2}} \frac{\mathrm{d}}{\mathrm{d} s}\left(r^{2} \kappa T_{0}^{5 / 2} \frac{\mathrm{d} T_{0}}{\mathrm{~d} s}\right)-\mathcal{R}_{0}\right]$.

The equilibrium pressure $p_{0}$ can be eliminated from Eq. (8) using the definition of the sound speed. The result is

$u_{0} \frac{\mathrm{d} u_{0}}{\mathrm{~d} s}=-\frac{c_{s}^{2}}{\gamma \rho_{0}} \frac{\mathrm{d} \rho_{0}}{\mathrm{~d} s}-\frac{1}{\gamma} \frac{\mathrm{d} c_{s}^{2}}{\mathrm{~d} s}-g+\mathcal{F}$.

The sum of the sources and sinks of energy is defined through Eq. (11). The explicit form of this term is given by Eq. (13) where it is expressed through temperature (sound speed), flow speed, and the body force. It shows that either $\mathcal{S}$ or $\mathcal{F}$ must be positive when the flow and the temperature are constant, i.e., either heating or body force are required to sustain the equilibrium flow against gravitational attraction. However, the balance between the body force $\mathcal{F}$, the source term $\mathcal{S}$ and the terms included in $\mathcal{S}$ through Eq. (11) has no influence on the behaviour of the Alfvénic perturbations.

The first term in the right-hand side of Eq. (12) can be substituted from Eq. (10). This results in the following expression for $\mathcal{S}$ in terms of the flow speed, the sound speed (or temperature), and the body force:

$\mathcal{S}=\frac{u_{0} \rho_{0}}{B_{0}}\left[\frac{1}{2} \frac{\mathrm{d} u_{0}^{2}}{\mathrm{~d} s}+\frac{1}{\gamma-1} \frac{\mathrm{d} c_{s}^{2}}{\mathrm{~d} s}+g-\mathcal{F}\right]$.

From Eq. (12) we obtain the following expressions for the equilibrium quantities in terms of the flow speed and temperature:

$\rho_{0}(s)=\rho_{0}(0) \frac{\lambda(0)}{\lambda(s)} \exp \left(-\int_{0}^{s} \frac{\mathrm{d} s}{\Lambda(s)}\right)$,

$p_{0}(s)=p_{0}(0) \exp \left(-\int_{0}^{s} \frac{\mathrm{d} s}{\Lambda(s)}\right)$,

$B_{0}(s)=B_{0}(0) \frac{u_{0}(s)}{u_{0}(0)} \frac{\rho(s)}{\rho(0)}$,

$c_{\mathrm{A}}(s)=c_{\mathrm{A}}(0) \frac{u_{0}(s)}{u_{0}(0)}\left(\frac{\rho(s)}{\rho(0)}\right)^{\frac{1}{2}}$,

where

$\lambda=\frac{c_{s}^{2}}{\gamma g}$ and $\Lambda=\lambda /\left(1+\frac{1}{2 g} \frac{\mathrm{d} u_{0}^{2}}{\mathrm{~d} s}-\frac{\mathcal{F}}{g}\right)$

represent the local pressure scale height in a force-free static equilibrium and the local pressure scale height in a steady state, respectively. The scale height $\lambda$ is determined by temperature. Variations in the scale height $\Lambda$ can be due to changes in the flow speed, temperature and the body force. Expressions (14)-(17) extend the well-known results for static equilibria derived by Roberts (2004) to steady states. In the special case of a forcefree isothermal atmosphere $(\gamma=1)$, the steady state solutions were derived by Taroyan (2011).

Alternatively, Eq. (12) can be integrated to express the sound speed and other equlibrium quantities in terms of the flow speed, $u_{0}$, the magnetic field, $B_{0}$, and the body force, $\mathcal{F}$ :

$$
\begin{aligned}
c_{s}^{2}(s)= & \frac{u_{0}(s) B_{0}(s)}{u_{0}(0) B_{0}(0)} \\
& \times\left(c_{s}^{2}(0)+\int_{0}^{s} \frac{\gamma u_{0}(0) B_{0}(0)}{u_{0}(\tilde{s}) B_{0}(\tilde{s})}\left[\mathcal{F}-g-u_{0} \frac{\mathrm{d} u_{0}}{\mathrm{~d} \tilde{s}}\right] \mathrm{d} \tilde{s}\right) .
\end{aligned}
$$

\subsection{Linearised equations for torsional perturbations}

Equations (1)-(5) can be linearised when small amplitude perturbations are considered. Incompressible torsional perturbations are governed by the equations:

$\frac{\rho_{0} r}{B_{0}} \frac{\partial v_{\theta}}{\partial t}+\frac{\partial}{\partial s}\left(\frac{\rho_{0} r u_{0}}{B_{0}} v_{\theta}\right)=\frac{1}{\mu_{0}} \frac{\partial}{\partial s}\left(r b_{\theta}\right)$,

$\frac{1}{r B_{0}} \frac{\partial b_{\theta}}{\partial t}+\frac{\partial}{\partial s}\left(\frac{u_{0}}{r B_{0}} b_{\theta}\right)=\frac{\partial}{\partial s}\left(\frac{v_{\theta}}{r}\right)$, 
Eqs. (19) and (20) can be Fourier analysed with respect to $t$ and presented in the following canonical form (Taroyan 2011):

$\frac{\mathrm{d} x}{\mathrm{~d} s}=\frac{1}{c_{\mathrm{A}}^{2}-u_{0}^{2}}\left(u_{0} C_{1} x+C_{2} z\right)$,
$\frac{\mathrm{d} z}{\mathrm{~d} s}=\frac{1}{c_{\mathrm{A}}^{2}-u_{0}^{2}}\left(c_{\mathrm{A}}^{2} C_{1} x+u_{0} C_{2} z\right)$,

where

$x=r \int_{-\infty}^{\infty} b_{\theta} \exp (\mathrm{i} \omega t) \mathrm{d} t, \quad z=B_{0} r \int_{-\infty}^{\infty} v_{\theta} \exp (\mathrm{i} \omega t) \mathrm{d} t$,

$C_{1}(\omega, s)=\frac{\mathrm{d} u_{0}}{\mathrm{~d} s}-\mathrm{i} \omega, \quad C_{2}(\omega, s)=c_{\mathrm{A}}^{2} \frac{\mathrm{d}}{\mathrm{d} s}\left(\frac{u_{0}}{c_{\mathrm{A}}^{2}}\right)-\mathrm{i} \omega$,

and $\omega$ is the complex frequency. Small amplitude perturbations generated at the footpoint by photospheric motions propagate along the flux tube. The propagation of torsional motions is governed by the set of Eqs. (21), (22). A driver usually excites transient driver specific perturbations in addition to the eigenmodes and those perturbations are not captured by the governing equations. In order to establish the stability of the steady equilibrium state with respect to arbitrary torsional motions, we have to examine the eigenmodes of the system. For simplicity, it is assumed that there are no variations in the azimuthal magnetic field at the footpoint. In other words, the filed lines are vertically anchored in the photosphere. The imposed condition $b_{\theta}(s=0, t)=0$ remains the same for the transformed variable: $x(s=0)=0$. Motions arise due to azimuthal twists of the footpoint which can be arbitrary. A twist will evolve along the tube disturbing the eigenmodes of the flux tube. Since the system (21), (22) is linear, $v_{\theta}$ at $s=0$ can be an arbitrary nontrivial function of omega corresponding to arbitrary azimuthal twists. A similar approach in different contexts has been applied by other authors (for example, Ruderman et al. 2010).

\section{Analytical results}

The governing Eqs. (21), (22) can be solved analytically for some special cases. These cases are treated separately in the present section.

\section{1. $B_{0}=$ const., $u_{0}=$ const.}

According to the equation of continuity (7), the density and the Alfvén speed are constant. The derivatives in expressions (24) become zero and Eqs. (21), (22) are reduced to a second order ODE with constant coefficients:

$\left(c_{\mathrm{A}}^{2}-u_{0}^{2}\right) \frac{\mathrm{d}^{2} x}{\mathrm{~d} s}+2 \mathrm{i} \omega u_{0} \frac{\mathrm{d} x}{\mathrm{~d} s}+\omega^{2} x=0$.

The general solution is

$x=a_{1} \exp \left(\frac{\mathrm{i} \omega s}{c_{\mathrm{A}}+u_{0}}\right)+a_{2} \exp \left(\frac{-\mathrm{i} \omega s}{c_{\mathrm{A}}-u_{0}}\right)$

where $a_{1}, a_{2}$ are arbitrary constants.

$$
\text { 3.2. } B_{0}=\text { const., } \frac{\mathrm{d} u_{0}}{\mathrm{ds}}=\text { const. }
$$

The condition $B_{0}=$ const. combined with the continuity equation implies $u_{0} / c_{\mathrm{A}}^{2}=$ const.. Therefore, $C_{1}=C_{2}=-\mathrm{i} \omega$.
Equations (21), (22) are reduced to a second order ordinary differential equation with variable coefficients:

$\frac{\mathrm{d}}{\mathrm{d} s}\left(\left[c_{\mathrm{A}}^{2}-u_{0}^{2}\right] \frac{\mathrm{d} x}{\mathrm{~d} s}\right)-\frac{\mathrm{d}}{\mathrm{d} s}\left(\left[\frac{\mathrm{d} u_{0}}{\mathrm{~d} s}-\mathrm{i} \omega\right] u_{0} x\right)+\mathrm{i} \omega \frac{\mathrm{d}}{\mathrm{d} s}\left(u_{0} x\right)+\omega^{2} x=0$,

We introduce a new variable $\tau$ :

$\tau=\frac{u_{0}^{2}}{c_{\mathrm{A}}^{2}} \quad$ so that $\quad \frac{\mathrm{d}}{\mathrm{d} s}=\frac{\mathrm{d}}{\mathrm{d} \tau} \frac{\mathrm{d} \tau}{\mathrm{d} s}=\frac{u_{0}}{c_{\mathrm{A}}^{2}} \frac{\mathrm{d} u_{0}}{\mathrm{~d} s} \frac{\mathrm{d}}{\mathrm{d} \tau}$.

In terms of the new variable $\tau$, Eq. (27) can be represented as a hypergeometric differential equation

$\tau(1-\tau) \frac{\mathrm{d}^{2} x}{\mathrm{~d} \tau^{2}}+(c-[a+b+1] \tau) \frac{\mathrm{d} x}{\mathrm{~d} \tau}-a b x=0$

with parameters

$a=b=1-\frac{\mathrm{i} \omega}{\mathrm{d} u_{0} / \mathrm{d} s}, c=1$.

The general solution of Eq. (29) is

$$
\begin{aligned}
x= & a_{1} \times{ }_{2} F_{1}(a, b ; a+b+1-c ; 1-\tau) \\
& +a_{2}(1-\tau)^{c-a-b}{ }_{2} F_{1}(c-b, c-a ; c-a-b+1 ; 1-\tau),
\end{aligned}
$$

where ${ }_{2} F_{1}$ is the hypergeometric function and $a_{1}, a_{2}$ are arbitrary constants (Abramowitz \& Stegun 1972). Note that the solution (31) is singular at $\tau=1$ because the real part of the exponent $c-a-b$ is negative.

\section{3. $T_{0}=$ const., $u_{0}=$ const., $\mathcal{F}=0$}

The magnetic field strength is no longer constant and changes in the cross sectional area are possible. Expressions (14)-(18) are simplified due to a constant sound speed and Eqs. (21), (22) are reduced to

$$
\frac{u_{0}^{2}-c_{\mathrm{A}}^{2}}{c_{\mathrm{A}}^{2}} \frac{\mathrm{d}}{\mathrm{d} s}\left(c_{\mathrm{A}}^{2} \frac{\mathrm{d} x}{\mathrm{~d} s}\right)+\left(\frac{u_{0}}{\lambda}-\mathrm{i} \omega\right)\left(-\mathrm{i} \omega x+2 u_{0} \frac{\mathrm{d} x}{\mathrm{~d} s}\right)=0 .
$$

Similar to the treatment in the preceding section, a new variable $\tau$ is introduced:

$\tau=\frac{u_{0}^{2}}{c_{\mathrm{A}}^{2}}$ and $\quad \frac{\mathrm{d}}{\mathrm{d} s}=\frac{\mathrm{d}}{\mathrm{d} \tau} \frac{\mathrm{d} \tau}{\mathrm{d} s}=\frac{\tau}{\lambda} \frac{\mathrm{d}}{\mathrm{d} \tau}$.

The change of variable leads to a hypergeometric Eq. (29). The solutions are expressed through the same hypergeometric functions (31) with different parameters:

$a=-\mathrm{i} \frac{\omega \lambda}{u_{0}}, b=a+1, c=0$.

\subsection{Unstable modes}

The obtained analytical results can be used to establish the presence of unstable modes. It would be instructive to find explicit expressions that reveal the dependence of the growth rate on various parameters.

Our first simplifying assumption is that the magnetic field strength is constant throughout. We also assume that the flow speed is a linear function for $0<s<L$. The solution is therefore given by Eq. (31). The flow is continuous at $s=L$ and constant for $s>L$. The corresponding solution for $s>L$ is expressed 
through Eq. (26). The first term of Eq. (26) represents propagation along the flow with phase speed $c_{\mathrm{A}}+u_{0}$. The second term represents propagation against the flow with phase speed $c_{\mathrm{A}}-u_{0}$. We set $a_{2}=0$ as there are no sources of propagation for $s>L$.

Note that the described equilibrium with constant magnetic field implies variable temperature, according to Eq. (12). In the present study specifying the force term and the temperature is not necessary as this has no influence on the behaviour of the Alfvén waves.

The analytical treatment is facilitated when a small parameter exists. Equations (21), (22) and the solution (31) suggest that such a small parameter could be $1-\tau$ which represents the difference between the flow and the Alfvén speeds. Hence our final assumption: $u_{0} \sim c_{\mathrm{A}}$.

The hypergeometric functions in Eq. (31) are expressed through the hypergeometric series (Abramowitz \& Stegun 1972):

${ }_{2} F_{1}(a, b ; c ; 1-\tau)=\sum_{n=0}^{\infty} \frac{(a)_{n}(b)_{n}}{(c)_{n}} \frac{(1-\tau)^{n}}{n !}$.

Because $1-\tau$ is small we can retain only the first term in the series. The general solution (31) in the interval $0<s<L$ is then approximated by

$$
x \approx a_{1}^{-}+a_{2}^{-}(1-\tau)^{c-a-b} .
$$

The coefficient $a_{2}^{-}$is expressed through $a_{1}^{-}$using the boundary condition $x(s=0)=0$. Substituting the expressions (30) for the parameters $a, b, c$ we obtain

$\frac{x}{a_{1}^{-}}=1-\left(\frac{1-\tau(s)}{1-\tau(0)}\right)^{\frac{2 i \omega}{\mu_{0}^{\prime}}-1}$ where $u_{0}^{\prime}=\frac{\mathrm{d} u_{0}}{\mathrm{~d} s}$

The counterpart $z$ is found from Eqs. (21) and (37):

$$
\frac{z}{a_{1}^{-}}=-u_{0}-u_{0}\left(1+\frac{i u_{0}^{\prime}}{\omega}\right)\left(\frac{1-\tau(s)}{1-\tau(0)}\right)^{\frac{2 i \omega}{u_{0}^{\prime}}-1} \text { for } 0<s<L .
$$

The solutions for $s>L$ are

$$
x=a_{1}^{+} \exp \left(\frac{\mathrm{i} \omega s}{c_{\mathrm{A}}+u_{0}}\right), z=-u_{0} x .
$$

The continuity of the solutions at $s=L$ gives the desired dispersion relation:

$$
\frac{x\left(L^{-}\right) z\left(L^{+}\right)-x\left(L^{+}\right) z\left(L^{-}\right)}{a_{1}^{-} a_{1}^{+}} \approx u_{0}\left(2+\frac{\mathrm{i} \omega}{u_{0}^{\prime}}\right)\left(\frac{1-\tau(s)}{1-\tau(0)}\right)^{\frac{2 \mathrm{i} \omega}{u_{0}^{\prime}}-1} \approx 0 .
$$

The complex frequency is therefore given by the following expression:

$\omega \approx 2 \mathrm{i} u_{0}^{\prime}$.

The real part of the frequency is small because the phase speed of the backward propagating wave, $c_{\mathrm{A}}-u_{0}$, is small.

Equation (41) provides a simple criterion for the instability: the imaginary part of the frequency is positive when the flow speed has a negative gradient in the interval $0<s<L$. The perturbations grow exponentially leading to an instability. In the case of a positive flow speed gradient, the perturbations generated at the footpoint are damped. Of course, an equilibrium with $B_{0}=$ const. is not a very good representation of the solar atmosphere where the magnetic field is known to change with height. On the other hand, the analytical treatment is facilitated. A steady state flux tube of constant cross-section is unstable if it contains a segment where the flow decelerates.

In summary, analytical solutions are derived in Sects. 3.1-3.3. The solutions are necessary to match the numerical solutions at the boundary $s=L$. Two of the solutions with a constant flow and a constant temperature/magnetic field are used for the subsequent numerical treatment. The analytical approach in Sect. 3.4 is based on a simplified model in order to facilitate the treatment. Only physically acceptable solutions are selected. The main purpose of this section is to find an analytical criterion for the instability. We find that the instability requires a negative gradient in the flow profile, i.e., deceleration along the flux tube. This finding is later confirmed numerically for certain particular cases.

\section{Numerical results}

The analytical treatment in the preceding section leads to a simple and transparent instability criterion (41). However the results are obtained when the magnetic field is constant and a small parameter $\tau$ exists. A numerical treatment is required for a more realistic solar atmospheric model with variable magnetic field.

We consider a vertically expanding flux tube along which all equilibrium quantities are continuous. The equilibrium field strength is a decreasing function of height in the interval $0<s<L$.

The continuity of the variable $x$ at the boundary $s=L$ follows from the continuity of the field lines. The following equation can be derived from the governing Eqs. (21), (22):

$\frac{\partial z}{\partial s}=-\mathrm{i} \omega x+\frac{\partial}{\partial s}\left(u_{0} x\right)$

The continuity of $z$ follows from Eq. (42). The shooting method is applied to find the eigenfrequencies. Linear torsional perturbations are driven at $s=0$ and the solutions at $s=L$ are obtained by numerically integrating the governing equations with a fourth order Runge-Kutta method. The obtained solutions are matched with the analytical solutions in $s>L$ and the resulting numerical algebraic equation is solved for $\omega$.

A boundary condition at $s=L$ must be specified through one of the solutions derived in Sect. 3. In the photosphere and chromosphere, the solar magnetic field is highly structured. At photospheric levels the field appears to be clumped into intense (1-2 kilogauss) bundles with diameters of a few hundred kilometers. The gas pressure inside these flux tubes is lower than the outside pressure, which provides the confining force. The gas pressure declines with increasing height, and the flux tubes necessarily expand (e.g., Hollweg 1981).

It has been argued that when the flux tubes fan out the average field strength is 5-10 Gauss in coronal holes, and some 50-100 Gauss in the active regions (Hollweg 1990). Using simultaneous photospheric and chromospheric magnetograms, no evidence of expansion was found by Zhang \& Zhang (2000). On the other hand, with spectropolarimeter data from the SOT, Tsuneta et al. (2008) estimated an upper limit area expansion for flux tubes between the photosphere and lower corona in the southern polar region of the Sun to be 345 . We separately consider rapid and moderate expansion of flux tubes in the context of the Alfvén instability. 


\subsection{Rapidly expanding flux tubes}

A set of boundary conditions describing rapid expansion is provided by the conditions $u_{0}, T_{0}=$ const. in the region $s>L$. The corresponding wave solutions are provided in Sect. 3.3. The magnetic field becomes an exponentially decreasing function of height and the speed of the decrease is determined by the inverse of the scale height. Provided the flow is sub-Alfvénic in the region $s>L$ there will be a point where the decreasing Alfvén speed becomes equal to the constant flow speed also known as the Alfvén point. Equations (31) and (34) show that in order to have a finite solution at the Alfvén point the constant $a_{2}$ must be zero. The solution in the upper region then becomes

$x=a_{1} \times{ }_{2} F_{1}\left(-\mathrm{i} \omega \frac{\lambda}{u_{0}}, 1-\mathrm{i} \omega \frac{\lambda}{u_{0}} ; 2-2 \mathrm{i} \omega \frac{\lambda}{u_{0}} ; 1-\frac{u_{0}^{2}}{c_{\mathrm{A}}^{2}(s)}\right)$,

where $\lambda$ is the constant scale height for $s>L$. The counterpart $z$ in the region $s>L$ is determined from Eqs. (42), (21), (28) using the differentiation formula for hypergeometric functions (Abramowitz \& Stegun 1972):

$$
\begin{aligned}
& z=\frac{a_{1}}{2} \cdot \frac{\lambda \mathrm{i} \omega}{1-\mathrm{i} \omega \lambda / u_{0}} \\
& \times\left[\left(1-\frac{u_{0}^{2}}{c_{\mathrm{A}}^{2}}\right) \times{ }_{2} F_{1}\left(1-\mathrm{i} \omega \frac{\lambda}{u_{0}}, 2-\mathrm{i} \omega \frac{\lambda}{u_{0}} ; 3-2 \mathrm{i} \omega \frac{\lambda}{u_{0}} ; 1-\frac{u_{0}^{2}}{c_{\mathrm{A}}^{2}}\right)\right. \\
& \left.+2 \times{ }_{2} F_{1}\left(-\mathrm{i} \omega \frac{\lambda}{u_{0}}, 1-\mathrm{i} \omega \frac{\lambda}{u_{0}} ; 2-2 \mathrm{i} \omega \frac{\lambda}{u_{0}} ; 1-\frac{u_{0}^{2}}{c_{\mathrm{A}}^{2}}\right)\right] .
\end{aligned}
$$

In the region $0<s<L$, the equilibrium quantities are functions of distance, $s$, flow speed, $u_{0}$, and temperature, $T_{0}$. In the following numerical results, distance and speed are normalised with respect to the scale height $\lambda(0)$ and the sound speed $c_{\mathrm{s}}(0)$. As an example, a sound speed of $c_{\mathrm{s}}(0)=7.5 \mathrm{~km} \mathrm{~s}^{-1}$ at a photospheric level of $s=0$ corresponds to a scale height of $\lambda(0)=125 \mathrm{~km}$. For simplicity, no body force is added in the momentum equation. The upflow in the region $s>L=2$ is maintained by a positive source term $\mathcal{S}$ due to heating. The density and the Alfvén speed are both decreasing functions above $s=L$ as a consequence of the prescribed constant flow and temperature profiles.

\subsubsection{Subsonic flows}

Figure 2 shows an equilibrium with rapid expansion where the normalised magnetic field decreases by a factor of 260 over a distance of $4 \lambda(0)$. The temperature is kept constant over a distance $4 \lambda(0)$. The equilibrium flow profile which results in rapid expansion remains subsonic and is shown in the upper panel of Fig. 2. Two different profiles of the Alfvén speed corresponding to different values of $c_{\mathrm{A}}(0)$ are plotted with dashed lines.

The real and imaginary frequencies are plotted as functions of the photospheric Alfvén speed, $c_{\mathrm{A}}(0)$, in the lower panels of Fig. 2. The real and imaginary parts of an eigenfrequency are shown with a same linestyle. There is a damped mode with zero frequency (dotted) and a mode with an imaginary frequency that remains positive until about $c_{\mathrm{A}}(0)=4$. Other modes are heavily damped and not shown. The presence of a growing mode indicates an instability. Figure 2 shows that the Alfvén instability may set in for subsonic flows and supersonic Alfvén speeds.

The purely damped does not oscillate. This mode could be a feature of the rapid tube expansion as it does not appear in the case of moderately expanding tubes (Sect. 4.2). Purely damped modes have been found in previous studies (for example, De Moortel \& Hood 1999).
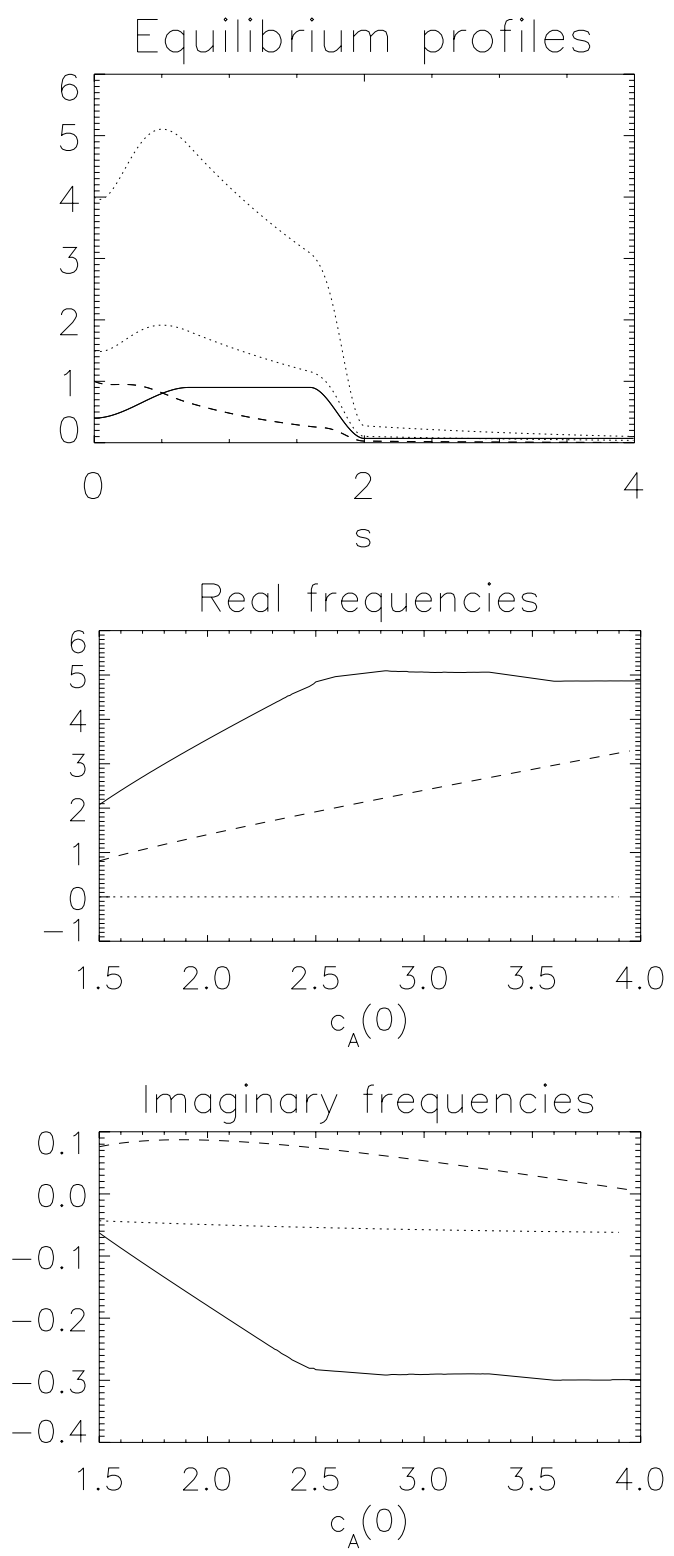

Fig. 2. Profiles of a subsonic equilibrium flow (solid) and a decreasing magnetic field (dashed). The segment of the tube with decelerating flow corresponds to rapid expansion of the flux tube. Smooth profiles of the Alfvén speed with $c_{\mathrm{A}}(0)=1.5$ and $c_{\mathrm{A}}(0)=4$ are plotted with dotted lines. The lower two panels display the continuous variation of $c_{\mathrm{A}}(0)$ from to 1.5 to 4 and the corresponding real and imaginary frequencies of the eigenmodes. The dashed lines represent an unstable mode. Strongly damped modes are not shown.

The mechanism of amplification is over-reflection and the mechanism of damping is partial reflection of the Alfvén waves in the region of negative flow gradient and rapid expansion. The process is discussed analytically in a simplified geometry by Taroyan (2008).

\subsubsection{Supersonic flows}

Another rapidly expanding flux tube is shown in the upper panel of Fig. 3. The magnetic field decreases by a factor of 120 over four scale heights $\lambda(0)$. The main difference with Fig. 2 is that the flow starts off supersonically and gradually becomes subsonic with height. The sound speed remains constant throughout. The lower panels show the presence of unstable modes for a range 


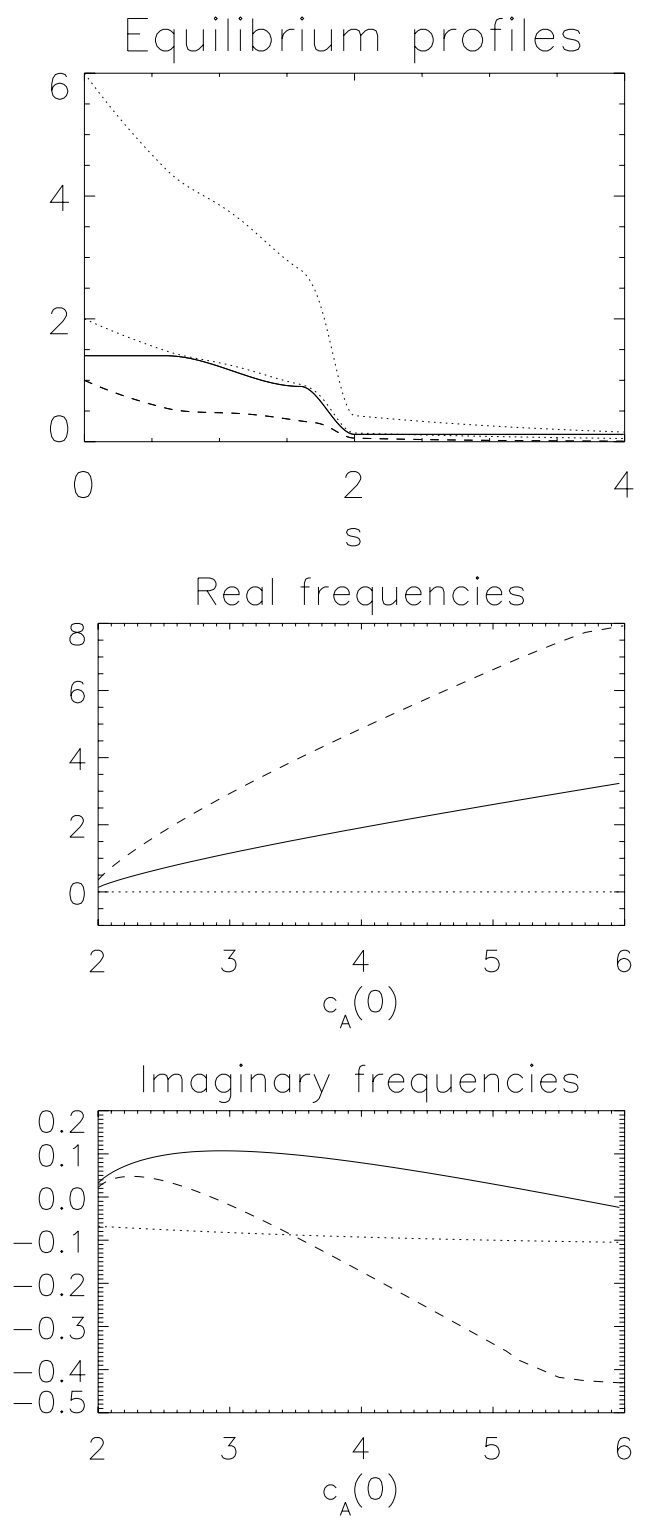

Fig. 3. Profile of a supersonic equilibrium flow (solid) corresponding to a rapidly expanding flux tube with decreasing magnetic field (dashed). Smooth profiles of the Alfvén speed for $c_{\mathrm{A}}(0)=2$ and $c_{\mathrm{A}}(0)=6$ are plotted with dotted lines. The lower two panels display the continuous variation of $c_{\mathrm{A}}(0)$ from to 2 to 6 and the corresponding real and imaginary frequencies of the eigenmodes. The solid and dashed lines correspond to unstable modes. Strongly damped modes are not shown.

of photospheric Alfvén speeds. Similar to the previous case, a purely damped mode is always present.

Figure 4 displays the dependence of the instability on the upflow speeds at the photospheric level, where an Alfvén speed of $c_{\mathrm{A}}(0)=3$ is set. Only flows with $u_{0}(0)<1.5$ are considered. For higher speeds the magnetic field becomes an increasing function of height. The upper panel of Fig. 4 shows two different equilibria with subsonic and supersonic flows at the photosphere. The lower panels show the presence of a purely damped mode and two modes which become unstable as the flow speed changes from $u_{0}(0)=0.5$ to $u_{0}(0)=1.5$.

The spatial structure of the real and imaginary parts of the eigenmodes $b_{\theta}$ is plotted in Fig. 5. The corresponding frequencies are plotted in Fig. 2. An Alfvén speed of $c_{\mathrm{A}}(0)=3$ is selected. The solid, dotted and dashed lines correspond to

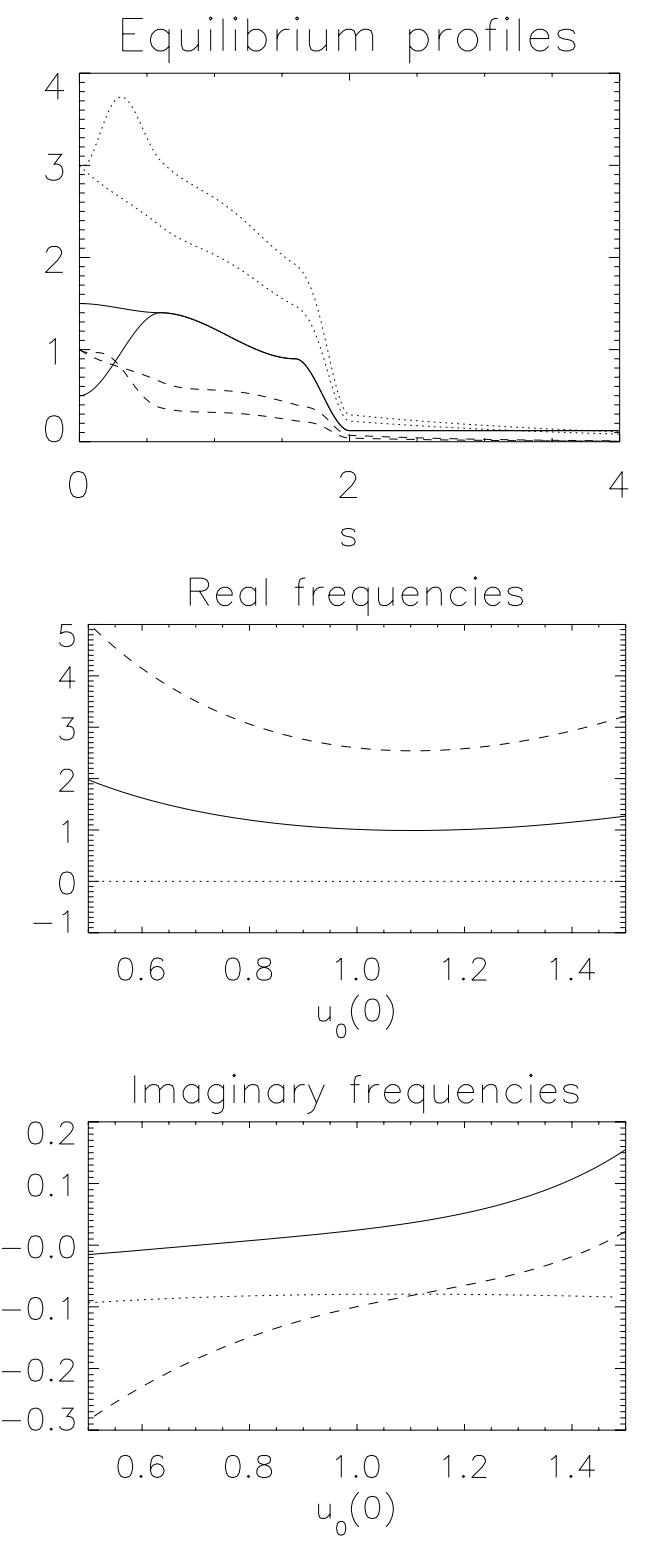

Fig. 4. Equilibrium flow profiles (solid) for a rapidly expanding tube with $u_{0}(0)=0.5$ and $u_{0}(0)=1.5$. The corresponding decreasing magnetic field is plotted with dashed lines and the profiles of the Alfvén speed are shown with dotted lines. For values of $u_{0}(0)$ higher than 1.5 the magnetic field is no longer a decreasing function of height. The lower two panels display the continuous variation of $u_{0}(0)$ from 0.5 to 1.5 and the corresponding real and imaginary frequencies of the eigenmodes. Unstable modes appear with increasing flow speed (solid and dashed lines).

$\omega_{r}=0,2.4,5$ and $\omega_{i}=-0.5,0.5,-0.3$, respectively. The variation in these frequencies with the Alfvén speed is plotted in Fig. 2. There is strong amplitude increase in the region of overreflection where the flow decelerates. In the upper region $s>2$, the amplitudes tend to decrease, but remain much higher than in the lower region. It is also worth noting that the amplitudes corresponding to modes with higher frequencies are larger.

\subsection{Moderately expanding flux tubes}

For moderately expanding tubes the flow and the magnetic field are constant in the upper region $s>L$. The solutions are given 

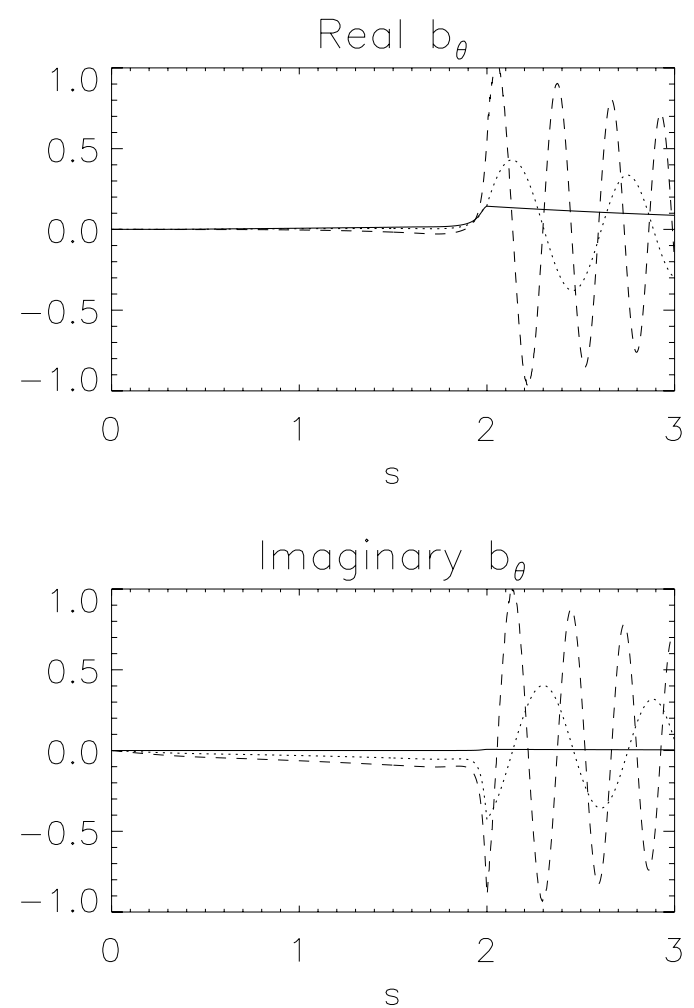

Fig. 5. Dependence of the eigenmodes on the spatial coordinate $s$ corresponding to Fig. 2. The Alfvén speed is fixed at $c_{\mathrm{A}}(0)=3$. The two panels display the continuous variation of the real and imaginary parts of the eigenmodes. The solid, dotted and dashed lines represent corresponding frequencies in Fig. 2. Strongly damped modes are not shown.

by the Eqs. (26), where $a_{2}=0$ is set as there are no sources of energy when $s>L$. The result is

$x=a_{1} \exp \left(\frac{\mathrm{i} \omega s}{c_{\mathrm{A}}+u_{0}}\right), z=-c_{\mathrm{A}}(L) x$.

At lower heights when $0<s<L$ the coefficients of the governing Eqs. (21), (22) are functions of height, the flow speed, and the magnetic field strength. The dispersion relation is solved by matching the numerical solutions in the region $0<s<L$ with the analytical solution in the region $s>L$.

The temperature profile depends on height, the flow speed, the magnetic field strength and the body force. Once $u_{0}$ and $B_{0}$ are fixed, there is no need to specify the body force. It will affect the temperature profile, the source term $\mathcal{S}$ and vice versa. However, the relationships between these quantities have no influence on the behaviour of the linear Alfvénic perturbations because the coefficients in the wave equations only depend on $u_{0}$ and $B_{0}$.

Firstly, we study the dependence of the instability on the deceleration of the flow along the tube. The equilibrium profiles and the corresponding eigenmode frequencies are plotted in Fig. 6. Similar to the case of rapidly expanding tubes, distance and speed are normalised with respect to the scale height $\lambda(0)$ and the sound speed $c_{\mathrm{s}}(0)$, respectively. The magnetic field is measured in arbitrary units. It decreases by a factor of 10 within a distance of 10 scale heights. As an example, a sound speed of $c_{\mathrm{s}}(0)=7.5 \mathrm{~km} \mathrm{~s}^{-1}$ at a photospheric level of $s=0$ corresponds to a scale height of $\lambda(0)=125 \mathrm{~km}$ and a reflection height of $10 \lambda(0)=1250 \mathrm{~km}$ : a pulse generated at $s=0$ propagates up and becomes either partially reflected, leading to damping, or over-reflected, leading to exponential growth and instability.
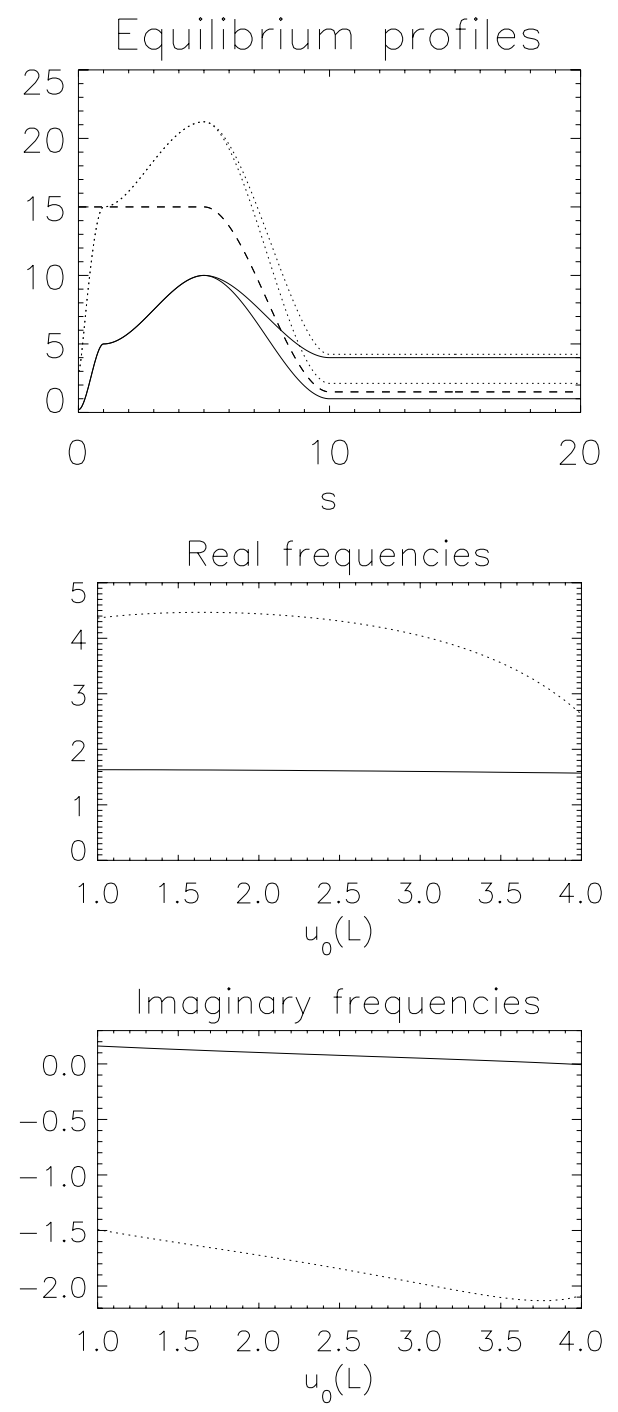

Fig. 6. Equilibrium flow profiles (solid) for a moderately expanding tube with $u_{0}(L)=1$ and $u_{0}(L)=4$. The corresponding magnetic field is plotted in arbitrary units with dashed lines and the profiles of the normalised Alfvén speed are shown with dotted lines. The lower two panels display the continuous variation of $u_{0}(L)$ from to 1 to 4 and the corresponding real and imaginary frequencies of the eigenmodes. An unstable mode (solid) is shown. The remaining modes are heavily damped. The real and imaginary parts of one such mode are plotted with a dotted lines.

A strong negative gradient in the flow favours the amplification of the waves.

Continuous equilibrium profiles with two different values of the flow speed at the interface $s=L\left(u_{0}(L)=1\right.$ and $u_{0}(L)=$ 4) are plotted in the top panel of Fig. 6. A zero level Alfvén speed of $c_{\mathrm{A}}(0)=3$ is chosen. The flow accelerates within a short distance from a photospheric value of $u_{0}(0)=0.2$ and continues to increase until a maximum of $u_{0}(s)=10$ is reached at a height of $s=5 \lambda(0)$. As a result, a maximum Alfvén speed of $c_{\mathrm{A}}=22$ is reached. The tube expands between $s=5$ and $s=10$ where the flow continues to remain sub-Alfvénic due to deceleration with height.

The corresponding variations of the real and imaginary frequencies between $u_{0}(L)=1$ and $u_{0}(L)=4$ are plotted in the two lower panels. An unstable mode represented by a solid line is present. The mode eventually becomes stable as the flow 


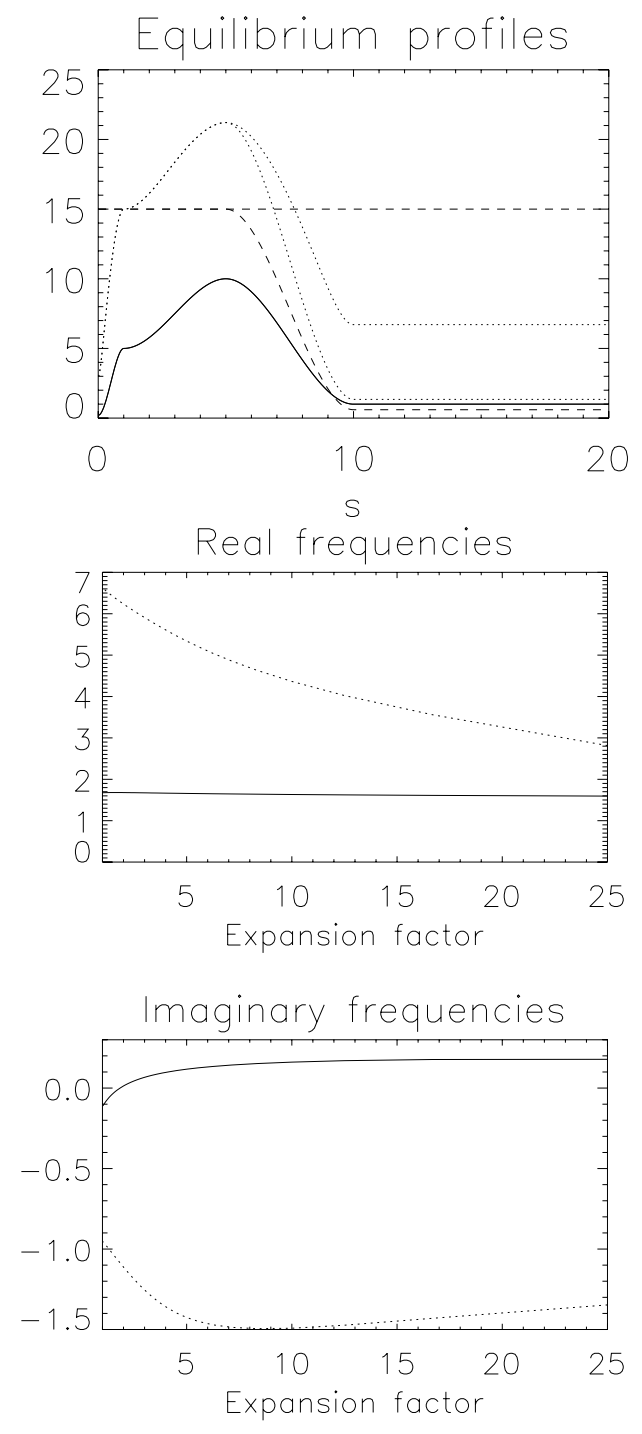

Fig. 7. Different magnetic field profiles for a moderately expanding tube are plotted in the upper panel with dashed lines. The first profile is constant, the second one decreases by a factor of 25 . The corresponding Alfvén speeds are plotted with dotted lines. The flow profile is fixed. The lower two panels display the eigenmode frequencies as functions of the expansion factor which is reciprocal to the magnetic field strength. An unstable mode appears as the tube begins to expand.

speed $u_{0}(L)$ becomes large. A strongly damped mode with a higher frequency is also plotted.

Finally, we study the dependence of the instability on the expansion factor of the flux tube which is reciprocal to the magnetic field ratio $B_{0}(L) / B_{0}(0)$. Two equilibria with different values of $B_{0}(L)$ are shown in the top panel of Fig. 7. The upper dashed line represents a flux tube with constant radius, the lower dashed line represents a tube with an expansion factor of 25 . The corresponding Alfvén speed profiles are plotted with dotted lines. In both cases, a photospheric value of $c_{\mathrm{A}}=3$ is selected. The flow profile is fixed with a photospheric speed of $u_{0}(0)=0.2$. An increase in the expansion factor leads to a decrease in the Alfvén speed $c_{\mathrm{A}}(L)$ as a consequence of the continuity equation.

Two eigenmode frequencies are displayed in Fig. 7. The first one plotted with a solid line has approximately constant frequency and becomes exponentially amplified is tubes with larger expansion factors. For comparison, a second mode with variable

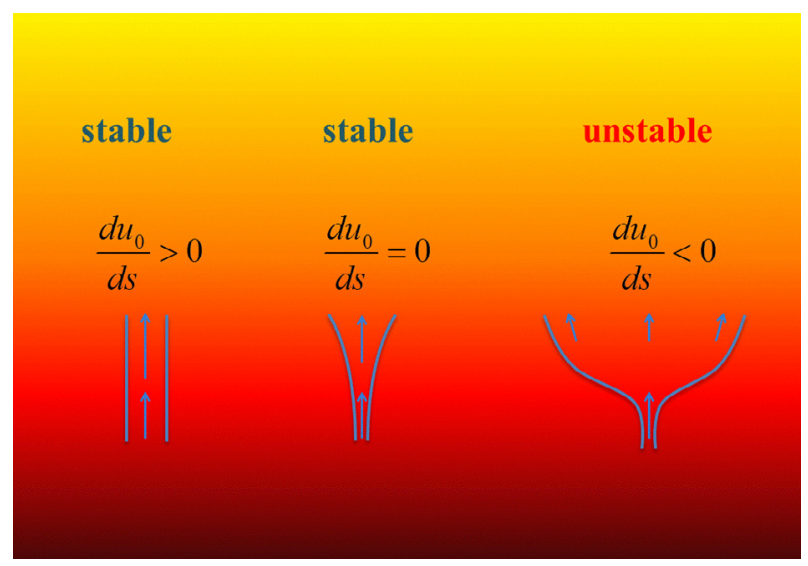

Fig. 8. Comparison of three tubes in a gravitationally stratified atmosphere. The arrows denote the flow speed. The first two tubes remain stable. The third tube is unstable due to rapid expansion and deceleration of the flow.

frequency and damping rate is plotted with a dotted line. The remaining strongly damped modes are not shown.

\section{Discussion and summary}

A vertical open magnetic flux tube in a gravitationally stratified atmosphere is considered. The tube is permeated by a vertical upflow driven by heating or a body force. Equilibria incorporating the effects of a vertical force, heating, and losses are derived analytically. Analytical solutions for torsional perturbations in the upper regions for different types of equilibria are derived. The governing equations for torsional motions are integrated with a fourth order Runge-Kutta method and matched with the analytical solutions in the upper regions to obtain a numerical dispersion relation which is solved for the eigenmode frequencies. Analytical treatment of the dispersion relation becomes possible when the flux tube has a constant cross-section. A steady state flux tube of constant cross-section is unstable if it contains a segment where the flow decelerates.

A numerical analysis is carried out for flux tubes with variable cross-sections which are permeated by subsonic or supersonic plasma flows. The results show that, in general, the instability favours lower Alfvén speeds and higher flow speeds at the photosphere. On the other hand, the instability is suppressed when the flow does not decelerate in the upper regions or when the flux tube does not expand. Figure 8 illustrates this by comparing three tubes two of which remain stable, whereas the third one is unstable due to expansion and deceleration of the flow.

The equilibrium is unstable due to the presence of Alfvénic perturbations. However the equilibrium does exist and it is analytically constructed in Sect. 2 . In the case of an initial static background the model has been tested by Hollweg et al. (1981) and others. In the linear regime, the equilibrium quantities derived in Eqs. (14)-(17) remain constant in time. Figures 2-7 show that the perturbations are damped when the negative flow gradient or the expansion factor are not large enough. The requirement of a negative flow gradient is somewhat similar to the requirement of a flow shear in the Kelvin-Helmholtz instability. Of course, the mechanisms are different.

The decelerating flow and the tube expansion essentially set up a cavity between the photosphere and the height of reflection/over-reflection. The generated torsional perturbations exponentially grow as they bounce back and forth within the cavity. It is important to emphasise that the amplifying cavity 
is different from resonant cavities discussed by where certain frequencies are required for growth and the growth itself is not necessarily exponential (Hollweg 1984; Matsumoto \& Shibata 2010). Instead, an arbitrary pulse will always grow exponentially as long as the equilibrium satisfies the instability criteria.

The amplified perturbations should subsequently couple to longitudinal shocks or dissipate their energy through another process as they become nonlinear. This may have important implications for solar atmospheric heating and wind acceleration.

We have already mentioned about the abundance of Alfvén waves in recent observations. An observational signature of the Alfvén instability would be the presence of standing or both upward and downward propagating large amplitude Alfvén waves in the lower solar atmosphere. Above the reflection height only upward propagating waves are expected to exist.

The equation of continuity (7) can be rewritten as $\frac{u_{0} B_{0}}{c_{\mathrm{A}}^{2}}=$ const. This shows that the tube expansion or the deceleration of the flow are both equivalent to a decreasing Alfvén speed. Hence over-reflection becomes possible when the Alfvén speed decreases in a section of the tube.

For example, according to Fig. 6, for a photospheric sound speed of $7.5 \mathrm{~km} \mathrm{~s}^{-1}$ and a flow speed of $u_{0}(0)=1.5 \mathrm{~km} \mathrm{~s}^{-1}$ the maximum Alfvén speed for which over-reflection still occurs is $30 \mathrm{~km} \mathrm{~s}^{-1}$ at a height of $1250 \mathrm{~km}$. The reported Alfvén speeds at those heights have similar values (Hollweg 1981).

The Alfvén speed is expected to be lower in spicular regions compared with nonspicular regions due to enhanced densities. On the other hand, higher Alfvén speeds would require unrealistically strong magnetic fields and high flow speeds at higher altitudes. It must be added that the present model considers equilibria that are unstable with respect to the Alfvénic perturbations. Therefore, a comparison between an unstable equilibrium and the physical parameters in the solar atmosphere is not very meaningful. Analysis of the nonlinear evolution of the instability is therefore required.

Another important question to address is the validity of a time-independent flow. This assumption can be justified if the periods of the waves are small compared with the lifetime of the flow. A typical lifetime of a spicule is $10-20 \mathrm{~min}$. On the other hand, the predicted periods of the unstable waves are less than a minute. The role of time-dependent flows should be addressed in the future.

\section{References}

Abramowitz, M., \& Stegun, I. A. 1972, Handbook of Mathematical Functions (Dover Publications)

Acheson, D. J. 1976, J. Fluid Mech., 77, 433

Antolin, P., \& Shibata, K. 2010, ApJ, 712, 494

Belien, A. J. C., Martens, P. C. H., \& Keppens, R. 1999, ApJ, 526, 478

De Moortel, I., \& Hood, A. 2003, A\&A, 408, 755

De Pontieu, B., Carlsson, M., Rouppe van der Voort, L. H. M., et al. 2012, ApJ, 752, L12

Fujita, Y., Suzuki, T., Kudoh, T., \& Yokoyama, T. 2007, ApJ, 659, L1

Gabriel, A. H. 1976, Phil. Trans. R. Soc. A, 281, 339

Jess, D. B., Mathioudakis, M., Erdélyi, R., et al. 2009, Science, 323, 1582

Hara, H., Watanabe, T., Harra L. K., et al. 2008, ApJ, 678, L67

Heyvartes, J., \& Priest, E. R. 1983, A\&A, 117, 220

Hollweg, J. 1981, Sol. Phys., 70, 25

Hollweg, J. 1984, Sol. Phys., 91, 269

Hollweg, J. 1990, Physics of Magnetic Flux Ropes, eds. E. R. Priest, \& L. C. Lee

Hollweg, J. V., Jackson, S., \& Galloway, D. 1982, Sol. Phys., 75, 35

Ionson, J. A. 1978, ApJ, 226, 650

Kudoh, T., \& Shibata, K. 1997, ApJ, 476, 632

Mariska, J. T., \& Hollweg, J. V. 1985, ApJ, 296, 746

Mathioudakis, M., Jess, D. B., \& Erdélyi, R. 2013, Space Sci. Rev., 175, 1

Matsumoto, T., \& Kitai, R. 2010, ApJ, 716, L19

Matsumoto, T., \& Shibata, K. 2010, ApJ, 710, 1857

McIntosh, S. W., Leamon, R. J., \& De Pontieu, B. 2011, ApJ, 727, 7

Moriyasu, S., Kudoh, T., Yokoyama, T., \& Shibata, K. 2004, ApJ, 601, L107

Morton, R. J., Verth, G., McLaughlin, J. A., \& Erdélyi, R. 2012, ApJ, 744, 5

Muller, R., Roudier, Th., Vigneau, J., \& Auffret, H. 1994, A\&A, 283, 232

Murawski, K., \& Musielak, Z. E. 2010, A\&A, 518, A37

Musielak, Z. E., Routh, S., \& Hammer, R. 2007, ApJ, 659, 650

Nakariakov, V. M., Ofman, L., Deluca, E. E., Roberts, B., \& Davila, J. M. 1999, Science, 285, 862

Ofman, L., \& Davila, J. M. 1995, J. Geophys. Res., 100, 23413

Parker, E. N. 1991, ApJ, 372, 719

Peter, H. 2000, A\&A, 360, 761

Roberts, B. 2004, ESA SP, 547, 1

Routh, S., Musielak, Z. E., \& Hammer, R. 2010, ApJ, 709, 1297

Ruderman, M. S., Robertson, D., \& Taroyan, Y. 2010, A\&A, 515, A33

Stenflo, J. O. 1989, A\&ARv, 1, 3

Sterling, A. C., \& Hollweg, J. V. 1984, ApJ, 285, 843

Sterling, A. C., \& Hollweg, J. V. 1988, ApJ, 387, 950

Taroyan, Y. 2008, Phys. Rev. Lett., 101, 245001

Taroyan, Y. 2009, ApJ, 694, 69

Taroyan, Y. 2011, A\&A, 533, A68

Tomczyk, S., McIntosh, S. W., Keil, S. L., et al. 2007, Science, 317, 1192

Tsuneta, S., Ichimoto, K., Katsukawa, Y., et al. 2008, ApJ, 688, 1374

Verth, G., Goossens, M., \& He, J.-S. 2011, ApJ, 733, L15

Xia, L.-D., Marsch, E., \& Curdt, W. 2003, A\&A, 399, L5

Zhang, H., \& Zhang, M. 2000, Sol. Phys., 196, 269 